\title{
Histoplasmosis en pacientes con SIDA sin manifestaciones cutáneo-mucosas
}

\author{
Fernando A. Messina ${ }^{1,2}$, Marcelo Corti, ${ }^{2,3}$, Ricardo Negroni', \\ Alicia Arechavala ${ }^{1}$, Mario Bianchi y Gabriela Santiso ${ }^{1}$
}

\footnotetext{
Unidad de Micología, Hospital de Enfermedades Infecciosas, Francisco J. Muñiz. Buenos Aires. Argentina.

2Departamento de Medicina Orientación Enfermedades Infecciosas, Facultad de Medicina, Universidad de Buenos Aires. Argentina.

${ }^{3}$ División B VIH-Sida, Hospital de Enfermedades Infecciosas Francisco J. Muñiz, Buenos Aires, Argentina.

Financiamiento: Ninguno. Conflicto de interés: Ninguno.

Recibido: 2 de septiembre de 2017

Aceptado: 2 de julio de 2018

Correspondencia a: Fernando Messina fmessina35@gmail.com
}

\begin{abstract}
Histoplasmosis in AIDS patients without tegumentary manifestations
Background: Histoplasmosis is a mycosis with a high prevalence in HIV/AIDS patients. Clinical presentation includes a wide spectrum of manifestations and diagnosis usually takes up to several weeks in patients who do not present cutaneous lesions. Aim: To determine the clinical and microbiological characteristics as well as some biochemical parameters in patients with AIDS-associated histoplasmosis without tegumentary lesions, in order to develop a guideline which enables an early empiric treatment in cases of difficult diagnosis. Methods: Medical records of 86 patients with histoplasmosis were reviewed; 31 patients with diagnosis of AIDS-associated histoplasmosis without cutaneous lesions were analyzed. Results: Fever was the most frequent symptom (96.7\%), lung involvement was observed in 22 patients $(70.9 \%)$, the most commonly radiological pattern was miliary pattern [(12/22), 54.5\%]. Nineteen patients presented with splenomegaly. Blood culture sensitivity was 93.3\% (28/30) and serology was positive only in $23.5 \%$ of the cases. Eight patients died $(25.8 \%)$. Patients in which CD4+ T cell lymphocytes count was $<50$ cells $/ \mu 1$, albumin levels $<2.5 \mathrm{~g} / \mathrm{dl}$ and who presented with pancytopenia had an unfavorable outcome. Conclusions: In HIV seropositive patients with fever associated to splenomegaly and bilateral miliar pattern in chest radiography, the empiric treatment with amphotericin B must be considered if signs and symptoms of unfavorable outcome are present and due to the time that it takes to arrive at an accurate diagnosis. In order to confirm the diagnosis, all microbiological samples should be collected prior to initiating therapy.

Keywords: Histoplasmosis argentina; AIDS; Histoplasma capsulatum; HIV.

Palabras clave: Histoplasmosis argentina; SIDA; Histoplasma capsulatum; VIH.
\end{abstract}

\section{Introducción}

L a histoplasmosis clásica es una micosis sistémica endémica, producida por el hongo dimorfo Histoplasma capsulatum var. capsulatum. Afecta al ser humano y a diversos animales. La mayoría de las primoinfecciones son asintomáticas o presentan manifestaciones respiratorias leves que se auto-limitan.

Las personas con diversos grados de compromiso de la inmunidad mediada por células tienen mayor riesgo de presentar formas graves de histoplasmosis diseminada cuyo curso es tanto más agudo y grave cuanto mayor sea el deterioro inmunológico.

La causa más común de histoplasmosis diseminada progresiva es la enfermedad avanzada debida al virus de la inmunodeficiencia humana (VIH). Desde 1987, se observó que más de $90 \%$ de los casos de histoplasmosis diseminada correspondían a enfermos con infección por VIH, lo que llevó a incluir a esta enfermedad como marcadora del SIDA ${ }^{1}$.

Histoplasma capsulatum es un hongo genéticamente heterogéneo; se han determinado 13 cariotipos y, según algunos autores, las disparidades genéticas observadas entre las cepas de Estados Unidos de América (E.U.A.) y las de América Latina, podrían ser responsables de las diferencias detectadas en las manifestaciones clínicas de esta micosis en ambas regiones endémicas ${ }^{2}$.

Hasta hace unos años se describían tres variedades del hongo, Histoplasma capsulatum var. capsulatum, Histoplasma capsulatum var. duboisii e Histoplasma capsulatum var. farciminosum, que se diferenciaban por las manifestaciones clínicas que presentaban los pacientes y por su distribución geográfica. En los últimos años, por las diferencias en las secuencias de su ADN, se han identificado ocho clados: 1) Norteamericana clase 1 clado $1 ; 2)$ Norteamericana clase 2 clado 2;3) Latinoamericana grupo A clado 3; 4) Latinoamericana grupo B clado 4; 5) Australiana clado 5; 6) Holandesa clado 6; 7) Eurasiática clado 7 y 8) Africana clado $8^{3}$.

La histoplasmosis es una afección de distribución geográfica muy amplia, ha sido descrita en los cinco continentes, con áreas de alta y baja endemicidad ${ }^{4}$.

Las lesiones cutáneo-mucosas son mucho más frecuentes en los enfermos nacidos en América del Sur ${ }^{5}$. Estas manifestaciones permiten que el diagnóstico se efectúe de forma precoz, porque suelen asociarse con la presencia de una gran cantidad de levaduras. 
El diagnóstico de las formas diseminadas agudas es el que ofrece mayores dificultades debido a su evolución rápida y a la ausencia de lesiones focales de fácil acceso para la biopsia o escarificación. Los hemocultivos dan resultados positivos en alrededor de 14-21 días, un plazo demasiado prolongado para confirmar el diagnóstico ${ }^{6}$.

En este trabajo retrospectivo se evaluaron las características clínicas y algunos parámetros bioquímicos de pacientes con enfermedad por VIH/SIDA que desarrollaron histoplasmosis aguda o subaguda sin manifestaciones tegumentarias, a fin de establecer pautas que permitirán emplear un tratamiento empírico y precoz que posibilitará disminuir la mortalidad y mejorar el pronóstico en aquellos casos de difícil diagnóstico.

\section{Objetivos}

Evaluar las características clínicas y la utilidad de algunos parámetros bioquímicos en pacientes con histoplasmosis aguda o subaguda sin manifestaciones tegumentarias.

\section{Métodos}

Se llevó a efecto un estudio descriptivo, retrospectivo y observacional en el que se analizaron las historias clínicas de 86 pacientes con diagnóstico de histoplasmosis asistidos en la Unidad Micología del Hospital de Referencia para Enfermedades Infecciosas del Gobierno de la Ciudad Autónoma de Buenos Aires F. J. Muñiz, en el período comprendido entre enero de 2010 y diciembre de 2012.

Se incluyeron pacientes con diagnóstico confirmado de histoplasmosis por cultivo o por la visualización de la fase levaduriforme del hongo en muestras clínicas observadas con tinciones especiales y microscopia óptica.

Se evaluaron las variables clínicas, de laboratorio, las co-morbilidades, los tratamientos recibidos y la mortalidad.

Se incluyeron pacientes con infección por VIH e histoplasmosis diseminada cuyo diagnóstico se realizó por el examen directo y cultivo de muestras de líquido cefalorraquídeo, esputo, lavado bronco-alveolar o por hemocultivo, mielocultivo o urocultivo. Se realizaron además pruebas serológicas para detección de anticuerpos anti-Histoplasma ${ }^{7}$.

Se excluyeron aquellos enfermos con diagnóstico de histoplasmosis obtenido por exámenes directos o cultivos de muestras de piel o mucosas, ya fuera mediante biopsias o por escarificación y los sujetos con serología negativa para el VIH.

\section{Análisis estadístico}

Se utilizó el programa Infostat 2011. Se calcularon medidas de valor central (media, mediana) y de dispersión (rango) y los porcentajes de positividad de los distintos parámetros evaluados. Se utilizó el test de proporciones para la comparación con los datos clínicos de los enfermos con lesiones cutáneas. Se consideró como significativa una diferencia de $\mathrm{p}<0,05$.

\section{Resultados}

En el período analizado se diagnosticaron 86 pacientes con histoplasmosis. Setenta y uno eran varones $(82,5 \%)$ y 15 mujeres $(17,4 \%)$. Se comprobaron 80 pacientes con coinfección por el VIH e histoplasmosis $(93,0 \%)$. Cuarenta y nueve $(61,3 \%)$ tenían manifestaciones en piel o mucosas.

Cumplieron con los criterios de inclusión 31 pacientes, por lo cual surge una prevalencia de pacientes con SIDA e histoplasmosis sin manifestaciones tegumentarias de $38,8 \%$.

La mediana de edad fue de 36 años (rango 18-60 años).

Once $(35,4 \%)$ enfermos conocieron su condición serológica frente al retrovirus en el momento del diagnóstico de la histoplasmosis.

Veinticinco pacientes $(80,6 \%)$ no recibían tratamiento con fármacos anti-retrovirales al momento de la primera consulta. La mediana de linfocitos (LT) $\mathrm{CD}^{+}$fue de 36 céls $/ \mu 1$ (rango 3-264 céls/ $\mu 1$ ). Veintitrés enfermos (74,2\%) tenían menos de $100 \mathrm{LT} \mathrm{CD}^{+} / \mu 1$ y sólo un paciente tuvo un valor de $264 \mathrm{LT} \mathrm{CD}^{+} / \mu 1$ en el momento del diagnóstico de la histoplasmosis.

La fiebre fue el síntoma más frecuente y se constató en treinta casos $(96,7 \%)$, en veintisiete $(87,0 \%)$ se comprobó pérdida de peso mayor a $10 \%$ de su valor habitual, doce $(38,7 \%)$ presentaron tos y once $(35,4 \%)$ disnea. Seis refirieron sudoración nocturna, cinco presentaron diarrea, en cuatro se constató la presencia de adenopatías cervicales, tres refirieron disfagia y un paciente presentó ictericia, equimosis, artralgias y cefalea. El compromiso pulmonar se comprobó en 22 enfermos (70,9\%); el patrón radiológico predominante fue el miliar o micronodulillar bilateral [(12/22 enfermos), 54,5\%]; el patrón retículonodulillar fue observado en seis pacientes y el compromiso intersticial se presentó sólo en cuatro. En nueve pacientes $(29,0 \%)$ la radiografía de tórax fue normal. En la Figura 1 se observa una radiografía de tórax con patrón micronodulillar.

Los hemocultivos por lisis-centrifugación fueron positivos en $28 / 30$ pacientes $(93,3 \%$ ), en enfermos sin lesiones cutáneo-mucosas. Sólo en un caso no se obtuvieron muestras para hemocultivos y el diagnóstico se obtuvo por cultivo del lavado bronco-alveolar. En los dos casos restantes los hemocultivos fueron negativos y el diagnóstico se alcanzó por cultivos del esputo en un caso y de la médula ósea en el otro.

Se examinaron 18 muestras de esputo; en dos desarrolló $H$. capsulatum en los medios de cultivo habituales y en uno de estos dos se visualizaron las levaduras en el examen directo mediante la coloración de Giemsa. El 
Figura 1. Radiografía de tórax frente con patrón microno dulillar.

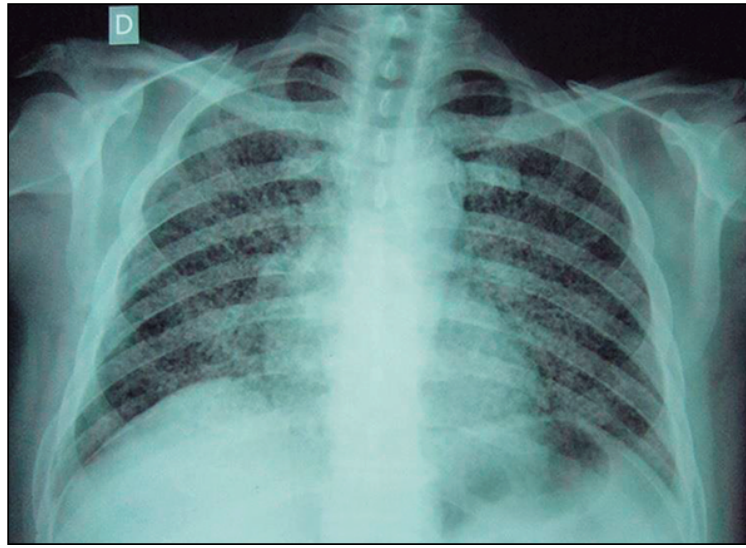

lavado bronco-alveolar se realizó en doce pacientes; se obtuvo desarrollo del hongo en diez muestras y en tres casos, además, se observaron levaduras en el examen directo.

Se efectuó punción aspiración de médula ósea con aguja fina en nueve casos, obteniéndose desarrollo en los cultivos en ocho pacientes; sin embargo, sólo se observaron levaduras en el examen directo en dos de

Tabla 1. Características de los enfermos con histoplasmosis sin lesiones cutáneo-mucosas

Pacientes sin lesiones de piel y/o mucosas $\mathrm{n}(\%)$

\begin{tabular}{lr} 
Número de pacientes & 31 \\
Sin tratamiento anti-retroviral & $25(80,6)$ \\
LT CD4 ${ }^{+}:>100$ céls/ml & $8(25,8)$ \\
LT CD $^{+}: 50$ - 100 céls/ml & $6(19,4)$ \\
LT CD4 $^{+}:$< 50 céls/ml & $17(54,8)$ \\
Fiebre & $30(96,8)$ \\
Lesiones de piel, examen directo positivo & 0 \\
Lesiones en mucosas, examen directo positivo & 0 \\
Diarrea & $5(16,1)$ \\
Oclusión intestinal & 0 \\
Anemia & $28(90,3)$ \\
Leucopenia & $19(61,3)$ \\
Plaquetopenia & $11(35,5)$ \\
Aumento de LDH & $19(61,3)$ \\
Aumento de transaminasas & $15(48,4)$ \\
VHS > 50 mm en ${ }^{\circ}{ }^{\prime}$ hora & $27(87,1)$ \\
Radiografía de tórax normal & $9(29,0)$ \\
Radiografía de tórax patrón micronodulillar (miliar) & $12(38,7)$ \\
Radiografía de tórax patrón intersticial & $4(12,9)$ \\
Radiografía de tórax patrón retículonodulillar & $6(19,4)$ \\
Ecografía abdominal normal & $5(18,5)$ \\
Esplenomegalia homogénea o ligeramente heterogénea & $19(70,4)$ \\
Adenopatías mesentéricas o precava & $14(51,9)$ \\
Pared colónica engrosada & $1(3,7)$ \\
Ascitis & $3(11,1)$ \\
Hepatomegalia & $13(48,1)$ \\
No se realizó ecografía abdominal & $4(12,9)$ \\
& \\
\hline
\end{tabular}

estas muestras. A un paciente se le realizó biopsia de médula ósea con presencia de levaduras características en el examen directo y cultivos positivos.

Se analizaron 17 muestras de suero para la detección de anticuerpos anti-H. capsulatum por las técnicas de inmunodifusión y contra-inmuno-electroforesis. La inmunodifusión fue positiva en dos casos y la contrainmuno-electroforesis en cuatro $(23,5 \%)$.

Se comprobaron diversas co-morbilidades asociadas con la histoplasmosis; seis pacientes presentaron candidiasis oral, cinco hepatitis B crónica y cuatro neumocistosis y tuberculosis. Es importante aclarar que en varios enfermos se comprobó más de una co-morbilidad.

Se realizó ecografía abdominal en 27 enfermos. En cinco pacientes no se observaron anormalidades. El hallazgo más característico fue la esplenomegalia observada en 19 pacientes $(70,4 \%)$, en diez ocasiones la víscera mostró características homogéneas y en nueve levemente heterogénea con el transductor de alta frecuencia y sin demostrar lesiones focales. El tamaño promedio del bazo fue de $148 \mathrm{~mm}$ de longitud (rango 130-184 mm). Se observaron adenomegalias retroperitoneales y mesentéricas en catorce enfermos $(51,8 \%)$. Se comprobó hepatomegalia en 13 casos (48\%) y fue siempre levemente heterogénea. Sólo tres enfermos presentaron ascitis, uno ileítis terminal y otro evidenció un aumento del espesor de la pared del colon derecho y del mesocolon.

La hipoalbuminemia resultó un hallazgo frecuente de laboratorio en esta serie; seis enfermos tenían concentraciones plasmáticas menores a $2,5 \mathrm{~g} / \mathrm{dl}$, cuatro de estos presentaron valores inferiores a $2 \mathrm{~g} / \mathrm{dl}$.

En siete enfermos se comprobaron concentraciones de la enzima láctico-deshidrogenasa (LDH) mayores a 1.000 UI/l; cuatro de ellos, presentaban además diagnóstico de neumocistosis.

Veinte pacientes $(64,5 \%)$ recibieron tratamiento inicial con anfotericina B desoxicolato en una dosis de $0,7 \mathrm{mg} /$ $\mathrm{kg} /$ día; seis enfermos $(19,3 \%)$ iniciaron el tratamiento con itraconazol $600 \mathrm{mg} /$ día durante las primeras $48 \mathrm{~h}$ y luego continuaron con el mismo fármaco, pero a dosis de $400 \mathrm{mg} /$ día. Cuatro pacientes habían fallecido al momento de obtenerse el diagnóstico y un paciente no realizó tratamiento porque se encontraba de alta cuando se llegó al diagnóstico de histoplasmosis y el servicio social no pudo localizarlo.

Durante el seguimiento fallecieron ocho pacientes $(25,8 \%)$ con histoplasmosis y SIDA, sin manifestaciones cutáneas. De los pacientes con manifestaciones cutáneas fallecieron igual cantidad, pero de un total de cuarenta y nueve casos $[(8 / 49) ; 16,32 \%]$. Veinte pacientes fueron dados de alta en buena condición clínica y tres se retiraron del nosocomio sin alta médica.

En la Tabla 1 se detallan las principales manifestaciones de los pacientes con histoplasmosis sin lesiones cutáneo-mucosas. 
En la Tabla 2 se describen los resultados de las pruebas microbiológicas de los pacientes con histoplasmosis sin compromiso tegumentario.

\section{Discusión}

En Sudamérica, incluyendo la Argentina, la histoplasmosis es una enfermedad sub-diagnosticada, a pesar de ser endémica y con alta prevalencia en pacientes inmunocomprometidos con síndrome febril ${ }^{8,9}$. En parte, este hecho se debe a que muchos de los síntomas son inespecíficos y se asemejan a los de la tuberculosis diseminada ${ }^{8}$ y a los de otras enfermedades que plantearían diagnósticos diferenciales alternativos, como micobacteriosis atípicas diseminadas y síndromes linfoproliferativos.

Las nuevas técnicas diagnósticas como la detección del antígeno galactomanano de $H$. capsulatum por ELISA en sangre y orina existen desde hace algunos años ${ }^{10}$, pero lamentablemente en la mayoría de los países subdesarrollados no están disponibles.

Las manifestaciones cutáneas o mucosas son muy frecuentes y permiten realizar un diagnóstico rápido mediante exámenes directos; en el período del estudio se comprobaron lesiones cutáneo-mucosas en $61 \%$ de los enfermos con SIDA e histoplasmosis, incidencia similar a la de otros países de Latinoamérica (38-85\%) y mayor a la observada en E.U.A. $(10 \%)^{11}$. En los pacientes no infectados por VIH, la frecuencia de lesiones mucocutáneas es similar ${ }^{12}$.

En aquellos enfermos que no presentan compromiso muco-cutáneo, el diagnóstico se confirma por el cultivo de secreciones respiratorias, mielocultivos, hemocultivos o, con menor frecuencia, por cultivo de líquido cefalorraquídeo. En todos estos casos el hongo puede tardar en desarrollar hasta 3 o 4 semanas $^{11,12,13}$, con lo cual el diagnóstico puede ser tardío. Por esta razón es muy importante conocer las características clínicas, los posibles resultados de los estudios por imágenes y los cambios en los parámetros bioquímicos, para establecer pautas que permitan implementar un tratamiento empírico en pacientes en los cuales la evolución del cuadro no permite esperar el diagnóstico de certeza. Antes de iniciar el tratamiento empírico deben tomarse todas las muestras necesarias para que en algún momento el tratamiento empírico pase a ser de certeza.

Entre los diagnósticos diferenciales se incluyen la tuberculosis diseminada, los linfomas y la leishmaniasis visceral. En la tuberculosis diseminada, la ultrasonografía o ecografía abdominal ayuda a sospechar el diagnóstico debido a que $95 \%$ de los enfermos presenta lesiones hipoecogénicas focales en el bazoy en un porcentaje similar se evidencian adenopatías retroperitoneales ${ }^{14}$. Después del sarcoma de Kaposi, los linfomas representan la neoplasia
Tabla 2. Resultados de pruebas microbiológicas realizadas en pacientes sin lesiones cutáneo-mucosas

\begin{tabular}{|c|c|}
\hline & $\begin{array}{c}\text { Pacientes sin lesiones } \\
\text { de piel y/o mucosas } \\
\mathrm{n}(\%)\end{array}$ \\
\hline Número de pacientes & 31 \\
\hline Hemocultivos $n$ & 30 \\
\hline Positivos & $28(93,3)$ \\
\hline Esputos $\mathrm{n}$ & 18 \\
\hline Directo positivo & $1(5,6)$ \\
\hline Cultivo positivo & $2(11,1)$ \\
\hline Lavado bronco-alveolar $\mathrm{n}$ & 14 \\
\hline Directo positivo & $3(21,4)$ \\
\hline Cultivo positivo & $10(71,4)$ \\
\hline Serologías $n$ & 17 \\
\hline CIE* positiva & $4(23,5)$ \\
\hline Inmunodifusión positiva & $2(11,8)$ \\
\hline Punción/biopsia de médula ósea $n$ & 10 \\
\hline Directo positivo & $2(20,0)$ \\
\hline Cultivo positivo & $8(80,0)$ \\
\hline Punción ganglionar $\mathrm{n}$ & 6 \\
\hline Directo positivo & $1(16,7)$ \\
\hline Cultivo positivo & $3(50,0)$ \\
\hline Diagnóstico dentro $48 \mathrm{~h}$ del ingreso & $1(3,2)$ \\
\hline Diagnóstico entre 2 y 7 días del ingreso & $3(9,7)$ \\
\hline Diagnóstico entre 7 y 14 días del ingreso & $5(16,1)$ \\
\hline Diagnóstico entre 14 y 21 días del ingreso & $11(35,5)$ \\
\hline Diagnóstico entre 21 y 28 días del ingreso & $11(35,5)$ \\
\hline Mortalidad & $(25,8)$ \\
\hline
\end{tabular}

más frecuente en los pacientes con SIDA. En este caso, el diagnóstico diferencial se plantea principalmente por la sintomatología sistémica, fiebre, sudores nocturnos y pérdida de peso (síntomas B ${ }^{15}$. La forma de presentación más frecuente de estos tumores en los pacientes con SIDA es la extranodal, con compromiso del tubo digestivo, la piel, la médula ósea y el sistema nervioso, como localizaciones más comunes.

Un estudio de autopsias de pacientes con infección por VIH con diagnóstico de histoplasmosis diseminada, permitió comprobar un elevado porcentaje de casos de compromiso del tracto gastrointestinal que alcanzó a $75 \%$ de los pacientes evaluados. En cambio, las manifestaciones clínicas del compromiso digestivo fueron mucho menos comunes ${ }^{16}$. Los métodos de diagnóstico por imágenes, estudios contrastados con bario o tomografía computarizada permiten observar la afectación segmenta- 
ria del colon, con estructuras rígidas, engrosamiento circunferencial de la pared del ciego y del colon ascendente y el estrechamiento del lumen, evidenciando, en algunos casos, el signo radiológico de "corazón de manzana" o de "manzana mordida", lo que obliga a realizar una biopsia intestinal, porque esta imagen también se observa en el cáncer de colon ${ }^{19}$.

Es importante incluir a la infección por citomegalovirus dentro de los diagnósticos diferenciales del compromiso del colon en pacientes con infección por VIH. En este caso se observa engrosamiento concéntrico de la pared del colon, pero en muy pocos casos presenta adenomegalias a ese nivel ${ }^{17}$.

El hallazgo de esplenomegalia es frecuente en los pacientes con histoplasmosis asociada con SIDA; ecográficamente se presenta como homogénea o levemente heterogénea ( $\sin$ lesiones focales) con los transductores de alta frecuencia ${ }^{17,18}$.

En la serie que se describe, todos los pacientes que presentaron serología positiva para $H$. capsulatum tuvieron buena evolución y la contra-inmuno-electroforesis demostró ser la técnica más sensible $(23,5 \%)$; sin embargo, al igual que en la mayoría de los estudios clínicos desarrollados en otros países, el rendimiento diagnóstico de estas técnicas en pacientes con SIDA es bajo ${ }^{19}$.

Los hemocultivos por lisis centrifugación han demostrado ser más sensibles que los realizados por métodos automatizados $^{11}$. Está demostrado que la sensibilidad de los hemocultivos está relacionada al volumen de sangre cultivada, que no debe ser inferior a $20 \mathrm{ml}^{20}$.

Otro hallazgo frecuente en esta serie fue la plaquetopenia, por lo común asociada a anemia y a leucopenia. En la serie que se analiza, la presencia de plaquetopenia se asoció con evolución desfavorable (6/11 enfermos fallecieron, 54,5\%). Algunos estudios mencionan, al igual que en nuestro trabajo, a la plaquetopenia como un factor de mal pronóstico ${ }^{21}$, pero en contraposición en otra publicación $^{22}$ no encontraron diferencias al comparar los enfermos con evolución favorable y los fallecidos. En cambio, este hallazgo sólo se comprobó en 6/49 pacientes con histoplasmosis y lesiones cutáneas $(\mathrm{p}=0,049)$.

En los pacientes incluidos en esta evaluación, aquellos con concentraciones séricas de albúmina inferiores a 2,5 g/ dl tuvieron peor pronóstico. De este subgrupo, fallecieron $5 / 6(83,33 \%)$. Este hallazgo es coincidente con lo descrito por la mayoría de los autores que señalan que enfermos con histoplasmosis diseminada e hipoalbuminemia tienen peor evolución y pronóstico ${ }^{23}$.

Otro marcador de mala evolución en la serie analizada fue el bajo recuento de $\mathrm{LT} \mathrm{CD}^{+}$; en este aspecto, siete de los ocho enfermos fallecidos tenían un recuento menor a 50 céls/ $\mu 1$.

Las transaminasas hepáticas estuvieron elevadas en menos de la mitad de los casos; en estos pacientes, la que se presentó con valores más altos fue la transaminasa glutámico-oxalacética (GOT).

La láctico-deshidrogenasa $(\mathrm{LDH})$ no resultó un parámetro de laboratorio de valor para sospechar el diagnóstico de histoplasmosis, ya que su aumento fue en general moderado y sólo se observaron concentraciones muy altas en aquellos sujetos con compromiso pulmonar concomitante por Pneumocystis jiroveci. A diferencia de lo señalado por otros autores ${ }^{24}$, las concentraciones de $\mathrm{LDH}$ dos veces por encima del valor considerado normal, no se relacionaron en este grupo de enfermos con un peor pronóstico, ya que sólo dos de los ocho pacientes fallecidos tenían concentraciones altas de LDH en sangre periférica.

En conclusión, el diagnóstico de histoplasmosis en pacientes con SIDA que no desarrollan compromiso muco-cutáneo se basa en un alto índice de sospecha en aquellos con manifestaciones clínicas de un síndrome infeccioso inespecífico asociado a compromiso respiratorio con patrón micronodulillar bilateral, esplenomegalia homogénea, pancitopenia y/o adenomegalias mesentéricas. La presencia de las características mencionadas, asociadas a los signos de mal pronóstico como la hipoalbuminemia y el recuento de LT CD4+ menor a 50 céls/ $\mu 1$ determinan evaluar la posibilidad de realizar un tratamiento empírico que luego podrá confirmarse con los hemocultivos y los cultivos de otras muestras obtenidas para estudios microbiológicos.

Es necesario contar con métodos de detección rápida de antígenos de $H$. capsulatum y con tratamientos de primera línea para mejorar el pronóstico de estos pacientes ${ }^{25}$.

\section{Resumen}

Antecedentes: La histoplasmosis es una micosis de gran relevancia en pacientes con SIDA. El cuadro clínico puede ser muy variado y, en enfermos que no desarrollan lesiones cutáneas, el diagnóstico suele demorar varias semanas. Objetivo: Establecer pautas que permitan emplear un tratamiento empírico y precoz en pacientes con histoplasmosis asociada al SIDA sin manifestaciones tegumentarias y determinar las características clínicas, microbiológicas y algunos parámetros bioquímicos en los casos de difícil diagnóstico. Métodos: Se analizaron las historias clínicas de 86 pacientes con histoplasmosis. Fueron seleccionados 31 enfermos con histoplasmosis asociada con SIDA sin lesiones cutáneas. Resultados: La fiebre fue el síntoma más frecuente $(96,7 \%)$, el compromiso pulmonar se comprobó en 22 enfermos (70,9\%). El patrón radiológico más frecuentemente hallado en las radiografías de tórax fue el miliar o micronodulillar bilateral [(12/22), 54,5\%]. Diecinueve enfermos presentaron esplenomegalia. Los hemocultivos demostraron una sen- 
sibilidad de $93,3 \%(28 / 30)$ y la serología fue positiva en $23,5 \%$ de los casos. Fallecieron ocho pacientes (25,8\%). Los pacientes con recuentos de linfocitos $\mathrm{T} \mathrm{CD} 4^{+}$menores a 50 céls $/ \mu 1$, albúmina menor a $2,5 \mathrm{~g} / \mathrm{dl}$ y pancitopenia evidenciaron un pronóstico desfavorable. Conclusiones: En los pacientes con infección por VIH, fiebre asociada a esplenomegalia y lesiones micronodulillares bilate- rales en la radiografía de tórax debe considerarse el tratamiento empírico con anfotericina B cuando existan signos o síntomas de mal pronóstico, debido al tiempo de demora hasta el diagnóstico definitivo. Previo a iniciar el tratamiento deben tomarse todas las muestras para los estudios microbiológicos que permitan confirmar luego la presunción diagnóstica.

\section{Referencias bibliográficas}

1.- Centers for Disease Control (CDC). Revision of the CDC surveillance case definition for acquired immunodeficiency syndrome. Morbid Mortal Wkly Rep MMWR 1987; 36: $1 \mathrm{~S}-15 \mathrm{~S}$.

2.- Canteros C E, Zuiani M S, Perrotta D E, Reyes-Montes MR, Granados J, Zúñiga $\mathrm{G}$, et al. Electrophoresis karyotype and chromosome-length polymorphism of Histoplasma capsulatum clinical isolates form Latin America. FEMS Immunol Med Microbiol 2005; 45: 423-8. DOI:10.1016/j. femsim.2005.05.015.

3.- Kasuga T, White T J, Koenig G, McEwen J, Restrepo A, Castañeda E, et al. Phylogeography of the fungal pathogen Histoplasma capsulatum. Mol Ecol 2003; 12: 3383-401. https://doi.org/10.1046/j.1365294X.2003.01995.x

4.- Adenis A, Aznar C, Couppié P. Histoplasmosis in HIV-infected patients: a review of new developments and remaining gaps. Curr Trop Med Rep 2014; 1: 119-28. DOI: 10.1007/ s40475-014-0017-8.

5.- Wheat L, Azar M, Bahr M, Spec A, Relich R, Hage C. Histoplasmosis. Infect Dis Clin North Am 2016; 30: 207-27. doi: 10.1016/j. idc.2015.10.009.

6.- Negroni R. Manifestaciones cutáneo-mucosas de la histoplasmosis. Dermatol Argent 2008; 14 (2): 104-12.

7.- Arechavala A, Robles A M, Negroni R, Bianchi M, Taborda A. Valor de los métodos directos e indirectos de diagnóstico en las micosis sistémicas asociadas al sida. Rev.Inst.Med. Trop. Sao Paulo 1993; 35(2):163-9.

8.- Nacher M, Adenis A, Aznar C, Blanchet D, Vantilcke V, Demar M, et al. How many have died from undiagnosed human immunodeficiency virus-associated histoplasmosis, A treatable disease? Time to act. Am J Trop Med Hyg 2014; 90: 193-4. doi: 10.4269/ajtmh.13-0226.

9.- Nacher M, Adenis A, Mc Donald S, Do
Socorro Mendonca Gomes M, Singh S, Lopes Lima I, et al. Disseminated histoplasmosis in HIV-infected patients in South America: A neglected killer continues on its rampage. PLOS Negl Trop Dis. 2013; 7: e2319. https:// doi.org/10.1371/journal.pntd.0002319

10.- Wheat L, Kohler R, Tewari R. Diagnosis of disseminated histoplasmosis by detection of Histoplasma capsulatum antigen in serum and urine specimen. N England J Med 1986; 314 : 83-8.

11.- Bianchi M, Negroni R. Estudio comparativo de dos sistemas de hemocultivos en micosis sistémicas asociadas al sida. Rev Argent Derm 1993; 74: 1-4.

12.- Kauffman C. Histoplasmosis. In: Dismukes W, Pappas PC, Sobel JD. Eds. Medical Mycology. Oxford University Press. 2003; p. 285-98.

13.- Kauffman C. Histoplasmosis a clinical and laboratory update. Clin Microbiol Rev 2007; 20: 115-32. DOI: 10.1128/CMR.00027-06.

14.- Giordani M T, Brunetti E, Brinazzi R, Benedetti P, Stecca C, Goblirsch, et al. Extrapulmonary mycobacterial infections in a cohort of HIV-positive patients: ultrasound experience from Vicenza, Italy. Infection 2013 41: 409-14. doi: 10.1007/s15010-012-0336-4.

15.- Corti M, Villafañe Fioti M F, Lewi D, Schtirbu R, Narbaitz M, de Dios Soler M. Linfoma del tubo digestivo y glándulas anexas en pacientes con SIDA. Acta Gastroenterol Latinoam 2006; 36: 190-6.

16.- Lamps L, Molina C, West B, Haggitt R C, Scott M A. The pathologic spectrum of gastrointestinal and hepatic histoplasmosis. Am J Pathol 2000; 113: 64-7. DOI:10.1309/X0Y2P3GY-TWE8-DM02.

17.- Méndez N. Aporte de la ecografía en el diagnóstico de la patología del tracto gastrointestinal en el paciente con SIDA. Rev Argent Infect Dr Francisco J Muñiz 2013; 16: 14-22.

18.- Escribano J, González J, Álvarez M, Rivero S, Raya J L, Ruza M. La ecografía abdominal en los pacientes con SIDA. Radiología 1998; 40 : 85-91.
19.- Quinet Leimann B, Vera Pizzini V, Medeiros Muniz M, Zancope Oliveira R. Histoplasmosis in a Brazilian center: clinical forms and laboratory tests. Rev Iberoam Micol 2005; 22: 141-6.

20.- Murray P, Witebsky F. The clinician and the microbiology laboratory. In: Mandell G, Bennett J, Dolin R. Eds. Principles and practice of Infectious Diseases. 2010. $7^{\circ} \mathrm{Ed}$. p. 233-65.

21.- Couppie P, Sobesky M, Aznar C, Bichat S, Clyti E, Bissuel F. Histoplasmosis and acquired immunodeficiency syndrome: a study of prognostic factors. Clin Infect Dis 2004; 38: 134-8. https://doi. org/10.1086/379770.

22.- Scarcelli Boigues B C, Miranda Paniago A M, Espindola Lima G M, Oliveira Nunes M, Oliveira Uehara S. Clinical outcomes and risk factors for death for disseminated histoplasmosis in patients with AIDS who visited a high-complexity hospital in Campo Grande, MS, Brazil. Rev Soc Bras Med Trop 2018; 51: 155-61. http://dx.doi. org/10.1590/0037-8682-0369-2017.

23.- Peigne V, Dromer F, Elie C, Lidove O, Lortholary O; French Mycosis Study Group. Imported acquired immunodeficiency syndrome-related histoplasmosis in Metropolitan France: a comparison of prehighly active anti-retroviral therapy and highly active anti-retroviral therapy eras. Am J Trop Med Hyg. 2011; 85: 934-41. doi: 10.4269/ ajtmh.2011.11-0224.

24.- de Francesco Daher E, de Sousa Barros F A, da Silva Júnior G B, Takeda C F, Mota R M, Ferreira M T, et al. Risk factors for death in acquired immunodeficiency syndromeassociated disseminated histoplasmosis. Am J Trop Med Hyg 2006; 74: 600-3.

25.- Adenis A, Nacher M, Hanf M, Vantilcke V, Boukhari R, Blachet D, et al. HIV-associated histoplasmosis early mortality and incidence trends: from neglect to priority. PLOS Negl Trop Dis 2014; 8: e3100. https://doi. org/10.1371/journal.pntd.0003100. 